\title{
Isotropic invariants of a completely symmetric third-order tensor
}

\author{
M. Olive ${ }^{1}$ and N. Auffray ${ }^{2}$ \\ 1) Université de Provence, Laboratoire d'Analyse, Topologie, Probabilités, \\ LATP UMR 7353 CNRS, 39 Rue F. Joliot-Curie, 13453 Marseille Cedex 13, \\ France $^{\mathrm{a})}$ \\ 2) Université Paris-Est, Laboratoire Modélisation et Simulation Multi-Echelle, \\ MSME UMR 8208 CNRS, 5 bd Descartes, 77454 Marne-la-Valle, \\ France $^{\mathrm{b})}$
}

In both theoretical and applied mechanics, the modeling of nonlinear constitutive relations of materials is a topic of prime importance. To properly formulate consistent constitutive laws some restrictions need to be impose on tensor functions. To that aim representations theorems for both isotropic and anisotropic functions have been extensively investigated since the middle of the XXth century. Nevertheless, in threedimensional physical space, most of the results are restricted to sets of tensors up to second-order. The purpose of the present paper is thus to get one step further and to provide an integrity basis for isotropic polynomial functions of a completely symmetric third-order tensor. To explicitly construct this basis, the link that exists between the $\mathrm{O}(3)$-action on harmonic tensors and the $\mathrm{SL}(2, \mathbb{C})$-action on the space of binary forms is exploited. We believe that such an integrity basis may found interesting applications both in continuum mechanics and in other fields of theoretical physics.

PACS numbers: 02.20.Qs, 62.20.D-

Keywords: Continuum Mechanics; Constitutive laws; Higher-order tensors; Integrity Basis; Classical Invariant Theory.

\footnotetext{
a)Electronic mail: marc.olive@math.cnrs.fr

b)Electronic mail: Nicolas.auffray@univ-mlv.fr
} 


\section{INTRODUCTION}

\section{A. Physical motivation}

The theory of representations for tensor functions is at the heart of the rational modeling of material behaviors ${ }^{6,39,41}$. Taking into account the different restriction a constitutive law must comply (material symmetry, material objectivity, ...) representation theorems provide the most general shape of tensorial functions satisfying all these constraints. Such a knowledge is important both from theoretical and experimental perspectives, since it indicates the number and the type of independent quantities to be observed in experience. A very interesting and sound review on topic has been written by Zheng ${ }^{41}$, hence we refer the reader to this publication for a deeper presentation of this subject.

In three-dimensional physical space, most of the results that are known today are restricted to sets of tensors up to second-order. In this publication we extend these result to the case of isotropic polynomial functions of a completely symmetric third-order tensor. This result is a first step towards a generalization of classical results to include third and higher-order tensors.

The motivation towards such a generalization is based on, at least, three physical needs:

1. To model non-linear constitutive relations for higher-order continua ${ }^{10,14,23,28,38}$. The isotropic hyper-elastic strain-gradient elasticity, for example, need to be supplemented by a non-linear constitutive relation between the hyper-stress tensor and the strain gradient tensor, both of them of third-order ${ }^{28}$;

2. To describe behaviors for anisotropic materials described by third-order structural ten$\operatorname{sors}^{5,27,41}$. To take anisotropy into account in the formulation of non-linear laws, the argument of the isotropic behavior is supplemented by some structural tensors, i.e. tensors that describe the material anisotropy. And, indeed, some material symmetry classes are described by higher-order structural tensors.

3. To identify the symmetry properties of a linear constitutive law experimentally identified in a non-optimal basis ${ }^{7}$. Expressed in a generic basis, it is difficult to identity the symmetry class of a linear operator, and to determine one of its optimal basis or representation. As studied, in a special case, for the elasticity tensor by Auffray et al. ${ }^{1}$ the 
study of polynomial relations between the elementary invariants of the tensor provide important information. To be extended to other behaviors, such as the piezoelectricity tensor (which is a third-order tensor), the first step is to know a set of elementary invariants of that object.

In the present paper, as a first step towards this goal, an integrity basis for isotropic polynomial functions of a completely symmetric third-order tensor is provided. The real vector space of these tensors will be denoted $\mathbb{T}_{(i j k)}$, the notation (..) indicates invariance under permutation of the indices in parentheses. This tensor space can be decomposed into a space of traceless completely symmetric third-order tensors $\left(\mathbb{H}^{3}\right)$ and a space of vectors $\left(\mathbb{H}^{1}\right)$. Contrary to $\mathbb{T}_{(i j k)}$ both $\mathbb{H}^{3}$ and $\mathbb{H}^{1}$ are $\mathrm{O}(3)$-irreducible spaces ${ }^{22,36}$. Hence, the integrity basis for isotropic polynomial functions for the space $\mathbb{T}_{(i j k)}$ is equivalent to the integrity basis for isotropic polynomial functions for the space $\mathbb{H}^{3} \oplus \mathbb{H}^{1}$. To make this paper as self-contained as possible, and to precisely state our result, some definitions need to be introduced.

\section{B. Some prior definitions}

An isotropic scalar-valued invariant function $W$ is formally defined by the property

$$
\forall \mathrm{T} \in \mathbb{T}_{(i j k)}, W(\mathrm{~T})=W(g \star \mathrm{T}), \forall g \in \mathrm{O}(3)
$$

in which the natural action of $\mathrm{O}(3)$ on $\mathbb{T}_{(i j k)}$ is denoted by $\star$ and defined by:

$$
\star: \mathrm{O}(3) \times \mathbb{T}_{(i j k)} \rightarrow \mathbb{T}_{(i j k)} ;(g, \mathrm{~T}) \mapsto g \star \mathrm{T} \text { with }(g \star \mathrm{T})_{i j k}:=g_{i p} g_{j q} g_{k r} T_{p q r}
$$

Two tensors $T_{1}$ and $T_{2}$ are said to be $\mathrm{O}(3)$-related, and denoted $\mathrm{T}_{1} \approx \mathrm{T}_{2}$, if there exists $g \in \mathrm{O}(3)$ such that $\mathrm{T}_{2}=g \star \mathrm{T}_{1}$. The set of all vectors $\mathrm{T} \in \mathbb{T}_{(i j k)}$ which are related to $\mathrm{T}_{1}$ by $\mathrm{O}(3)$ is called the $\mathrm{O}(3)$-orbit of $T_{1}$ and is denoted by

$$
\mathrm{O}(3) \star \mathrm{T}_{1}:=\left\{\mathrm{T}=g \star \mathrm{T}_{1} \mid g \in \mathrm{O}(3)\right\}
$$

Hence, as it can directly be observed, isotropic invariant functions are constant on $\mathrm{O}(3)$ orbits. Now, among all functions, let us consider more specifically the polynomial ones. As well-known from invariant theory, since the orthogonal Lie group $\mathrm{O}(3)$ is compact, the algebra of invariant polynomial functions on $\mathbb{T}_{(i j k)}$ is finitely generated ${ }^{4331}$ and, furthermore, in the 
real case, polynomial invariants separate the orbits. From now on, $G$ will either be $\mathrm{O}(3)$ or $\mathrm{SO}(3)$. A basis for the $G$-invariant polynomial algebra is called an integrity basis ${ }^{44}$ :

Definition I.1. Let $\mathbb{V}$ be a real vector space with a $G$-action. A finite set $p_{1}, \ldots, p_{k}$ of $G$-invariant polynomials on $\mathbb{V}$ is called an integrity basis if every $G$-invariant polynomial on $\mathbb{V}$ can be written as a polynomial in $p_{1}, \ldots, p_{k}$.

An integrity basis is said to be irreducible if none of its elements can be expressed as a polynomial of the others. It is worth noting that this definition does not exclude that some polynomial relations exist between generators. Such relations, which can not be avoided in most cases, are known as syzygies and their determination is a difficult problem.

Beside integrity bases, functional bases ${ }^{5,7,40}$ can be defined:

Definition I.2. Let $\mathbb{V}$ be a real vector space with a $G$-action. A finite set $s_{1}, \ldots, s_{k}$ of $G$-invariant functions of $\mathbb{V}$ is called a functional basis if

$$
s_{i}\left(v_{1}\right)=s_{i}\left(v_{2}\right), \forall i=1, \ldots, n
$$

implies $v_{1}=g \cdot v_{2}$ for some $g \in G$.

A functional basis is said to be irreducible if none of its elements can be expressed as a function of the others. It is worth noting that this definition does not preclude that some functional relations between generators exist. In the definition of a functional basis, basis invariants are not required to be polynomial. However for physical applications it is often more convenient to determine polynomial functional bases ${ }^{5,6}$.

Before going any further, the two aforementioned definitions have to be discussed. While the former is centered on finding a generating system for the algebra of $G$-invariant polynomial functions, the latter is concerned with the determination of a separating system, i.e. on finding a set of (polynomial) functions that separates $G$-orbits of $\mathbb{V}$ elements. This distinction is important because, although the algebra of invariant polynomials separates the orbits, this set might be very large. As a consequence, an integrity basis is a functional basis, but the converse is generally false ${ }^{7,40}$. Hence, the cardinal of a minimal integrity basis is generally greater than that of a functional basis. In mathematics, an irreducible functional basis is 
called a separating set ${ }^{11}$, but if their conciseness is appealing, no general algorithm currently exists to produce them. Let us now do a quick review on the state-of-the-art in invariant functions modeling in continuum mechanics.

\section{State-of-the-art in applied invariant theory}

Integrity and functional bases are currently known for invariant functions of an arbitrary number of vectors and skew and symmetric second order tensors ${ }^{6,27,41}$, that is for sets of tensors up to second-order. For higher-order tensors results are very partial and restricted to particular cases ${ }^{45}$. The reason lies in the fact that the classical geometrical methods used for low-order tensors cease to function since third-order tensors. Even if not directly expressed in these terms this point was clear to authors who worked on this topic ${ }^{7,33,34}$. As a consequence, for higher-order tensors, methods stemming from the classical invariant theory have to be employed. This change of point of view has important implications:

1. Due to the fact that, for sets of tensors up to second-order, with some geometric intuition functional bases can be constructed results mostly concern the constructions of such bases. For higher-order tensors this inductive procedure cannot be employed anymore, and attention has moved to integrity bases. This point is clear in the late works of Boehler ${ }^{7}$ and Smith $^{33}$.

2. If up to second-order, whole tensors can be considered as the elementary variables of isotropic functions, this point of view cannot be extended. Instead, tensor have to be decomposed into $\mathrm{O}(3)$-irreducible elements, that is, into a sum of completely symmetric traceless tensors. This decomposition is sometimes referred to as the harmonic decomposition ${ }^{15,22}$. $\mathrm{O}(3)$-irreducible tensors are the elementary variables of isotropic functions ${ }^{46}$.

The problem we are presently interested in concerns an extension of a result previously obtained by Smith and $\mathrm{Bao}^{33}$. In this reference the authors provide an integrity basis for isotropic functions of a traceless symmetric third-order tensor $\left(T \in \mathbb{H}^{3}\right)$. In our present paper we extend this result to isotropic functions of a full symmetric third-order tensor. In terms of tensor space, this amounts to consider a space constituted of a traceless completely 
symmetric third-order tensor and a vector, i.e. the tensor space $\mathbb{H}^{3} \oplus \mathbb{H}^{1}$. As said in this first part of the introduction, this result might found interesting applications in continuum mechanics to construct constitutive laws $6,10,13,41$.

Let us now briefly draw the big picture of the approach used to determine an integrity basis for $\mathbb{T}_{(i j k)}$.

\section{Technical construction}

There exists a deep link between the $\mathrm{SO}(3)$-action $^{47}$ on harmonic tensors and the $\mathrm{SL}(2, \mathbb{C})$ action on the space of binary forms, i.e. the space of complex homogeneous polynomials in $x, y$. This connection was already known by authors in mechanics ${ }^{7,33,35,41}$ but, except in few references ${ }^{7,33}$, has not really been exploited. In Boehler et al. ${ }^{7}$, for example, to obtain an integrity basis for a fourth-order completely symmetric traceless tensor $\left(\mathrm{T} \in \mathbb{H}^{4}\right)$ the authors used some purely mathematical results obtained by Shioda ${ }^{32}$. This work was about the construction of an integrity basis for $\mathrm{S}_{8}$, the space of binary forms of degree 8 under $\mathrm{SL}(2, \mathbb{C})$-action. Hence, the problem of the determination of integrity bases for tensor spaces can be rephrased in terms of binary forms. Such a reformulation allows one to reinvest existing tools from classical invariant theory. This strategy is adopted in the present paper.

The most famous approach was initiated by Hilbert ${ }^{21}$, and successfully applied (without any computer assistance) by Shioda ${ }^{32}$. More recently, and with the extensive use of computer, Dixmier and Lazard ${ }^{12}$, Bedratyuk ${ }^{4}$ and Popovisciu and Brouwer ${ }^{8,9}$ have derived integrity basis for binary forms up to $\mathrm{S}_{10}$, which would correspond to $\mathbb{H}^{5}$, i.e. fifth-order completely symmetric and traceless tensor space. It has to be emphasized that this approach relies on very intensive computations since matrix ranks have to be tested up to order 20000 . According to a mathematical point of view, this is essentially an algebraical geometric method that relies on the subtle notion of system of parameters of an algebra ${ }^{37}$. It appears that this notion is not an effective one: up to our knowledge, there is no general algorithm to decide whether a set of variables is a system of parameters or not. Instead we decide to use a nineteenth century algorithm first given by Gordan in $1868^{18}$. This approach leads to the constructive theorem IV.2 used in the present paper. 


\section{RESULTS}

In this section our main results are summed-up, and proofs postponed to the next sections. First let us consider the result obtained by Bao and Smith ${ }^{33}$. Their result will be given using the diagrammatic representation already used by Boehler et al. ${ }^{7}$.

Theorem II.1. An integrity basis for $\mathbb{R}\left[\mathbb{H}^{3}\right]^{\mathrm{O}(3)}$ is given by $I_{2}, I_{4}, I_{6}$ and $I_{10}$
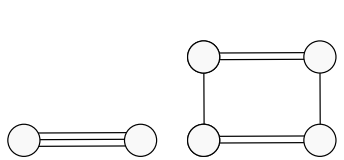

$I_{2}$

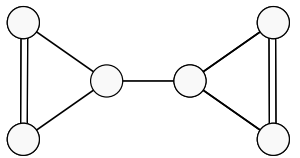

$I_{6}$

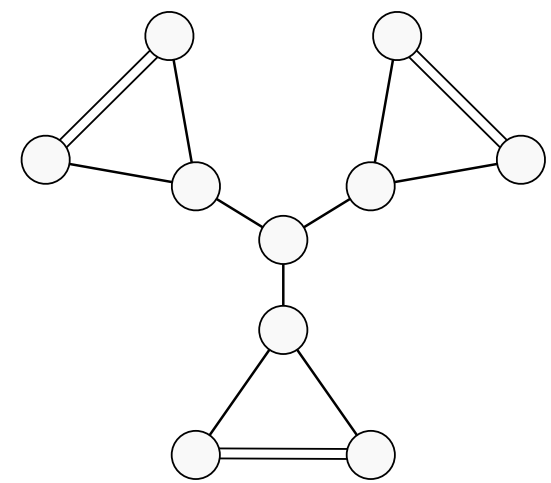

$I_{10}$

In this representation the big circle represents $\mathrm{D} \in \mathbb{H}^{3}$ and the lines index contractions. For instance, the invariant ${ }^{48} \lambda=\mathrm{D}_{i j k} \mathrm{D}_{i j k}$ has the graphical representation:

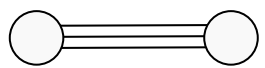

Now let us consider the case of a completely symmetric third order tensor. This situation is amount to add a vector $u \in \mathbb{H}^{1}$ to the previous component $D \in \mathbb{H}^{3}$. In the diagrammatic notation this vector component will be denoted by a small black dot. Hence, our main result is:

Theorem II.2. An integrity basis for $\mathbb{R}\left[\mathbb{H}^{3} \oplus \mathbb{H}^{1}\right]^{\mathrm{O}(3)}$ is given by:

$$
\begin{array}{lll}
I_{2}:=\mathrm{D}_{i j k} \mathrm{D}_{i j k} & J_{2}:=\mathrm{u}_{i}^{2} & I_{4}:=\mathrm{D}_{i j k} \mathrm{D}_{i j l} \mathrm{D}_{p q k} \mathrm{D}_{p q l} \\
J_{4}:=\mathrm{D}_{i j k} \mathrm{u}_{k} \mathrm{D}_{i j l} \mathrm{u}_{l} & K_{4}:=\mathrm{D}_{i j k} \mathrm{D}_{i j l} \mathrm{D}_{k l p} \mathrm{u}_{p} & L_{4}:=\mathrm{D}_{i j k} \mathrm{u}_{k} \mathrm{u}_{j} \mathrm{u}_{i} \\
I_{6}:=\mathrm{v}_{i}^{2} & J_{6}:=\mathrm{D}_{i j k} \mathrm{D}_{i j l} \mathrm{u}_{k} \mathrm{D}_{l p q} \mathrm{u}_{p} \mathrm{u}_{q} & K_{6}:=\mathrm{v}_{k} \mathrm{w}_{k} \\
L_{6}:=\mathrm{D}_{i j k} \mathrm{D}_{i j l} \mathrm{D}_{k} v_{l} & M_{6}:=\mathrm{D}_{i j k} \mathrm{D}_{p q k} \mathrm{u}_{i} \mathrm{u}_{j} \mathrm{u}_{p} \mathrm{u}_{q} & I_{8}:=\mathrm{D}_{i j k} \mathrm{D}_{i j l} \mathrm{u}_{k} \mathrm{D}_{p q l} \mathrm{D}_{p q r} \mathrm{v}_{r} \\
I_{10}:=\mathrm{D}_{i j k} \mathrm{v}_{i} \mathrm{v}_{j} \mathrm{v}_{k} & &
\end{array}
$$


in which
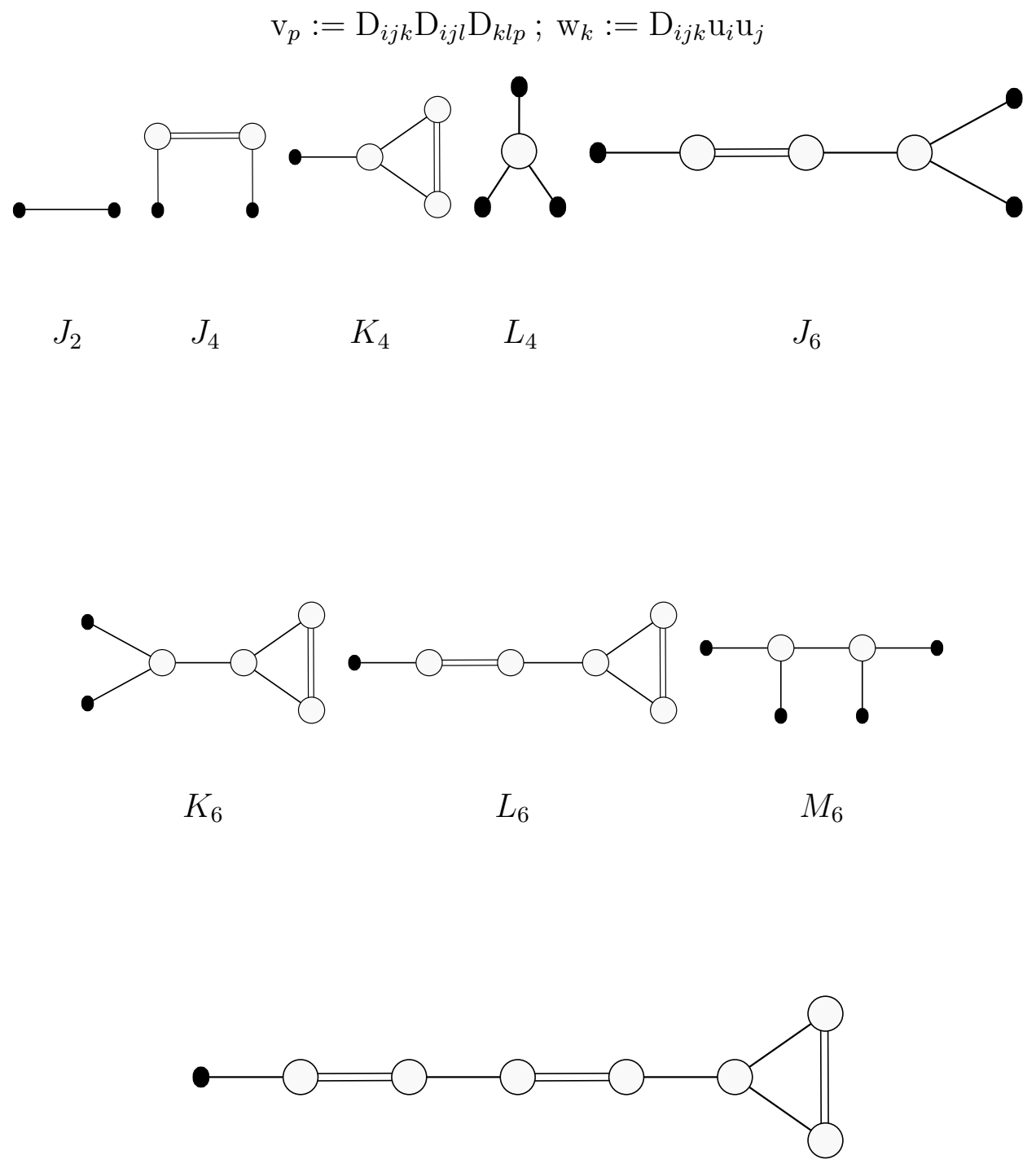

$I_{8}$

\section{MATHEMATICAL FRAMEWORK}

A. $\mathrm{O}(3)$-tensor spaces

The space $\mathbb{T}_{(i j k)}$ is endowed with the natural $\mathrm{O}(3)$-action given by I.2:

$$
(g \star \mathrm{T})_{i j k}:=g_{i l} g_{j m} g_{k n} \mathrm{~T}_{l m n}
$$

More generally, this action, sometimes referred to as the Rayleigh action, can be defined on any $k$ th-order tensor space $\mathbb{T}$. A subspace $\mathbb{F} \subseteq \mathbb{T}$ is said to be $\mathrm{O}(3)$-stable provided $g \cdot \mathbb{F} \subseteq \mathbb{F}$ 
for every $g \in \mathrm{O}(3)$. As can be observed $\mathbb{T}_{(i j k)} \subseteq \mathbb{T}_{i j k}$ is stable but can still be decomposed into smaller stable subspaces. In other terms $\mathbb{T}_{(i j k)}$ is not irreducible. As it will be detailed, $\mathrm{O}(3)$-irreducible tensors are encoded by harmonic tensors.

Let us consider $\mathbb{H}^{k}$ to be the space of $k$ th-order harmonic tensors ${ }^{15}$. The denomination harmonic is due to a classical isomorphism ${ }^{3}$ in $\mathbb{R}^{3}$ between $\mathbb{H}^{k}$ and the space of $k$ th-degree harmonic polynomials ${ }^{49}$. A classical mathematical result ${ }^{36}$ states that $\mathrm{SO}(3)$-action on $\mathbb{H}^{k}$ is irreducible: non-trivial $\mathrm{SO}(3)$-stable subspace ${ }^{50}$ cannot be found in $\mathbb{H}^{k}$. Now, it is easy to show that $\mathrm{O}(3)$-action on each $\mathbb{H}^{k}$ is irreducible. Furthermore, since $\mathrm{O}(3)$ is compact, the Peter-Weyl theorem ${ }^{36}$ ensures that every $\mathrm{O}(3)$-space can be isomorphically decomposed into irreducible subspaces. This decomposition is sometimes referred to as the harmonic decomposition ${ }^{15,25}$.

The space $\mathbb{T}_{(i j k)}$ can be uniquely decomposed ${ }^{51}$

$$
\mathbb{T}_{(i j k)} \simeq \mathbb{H}^{3} \oplus \mathbb{H}^{1}
$$

i.e. there exists an isomorphism

$$
\mathrm{T} \mapsto(\mathrm{D}, \mathrm{u}) \text { with } \mathrm{D} \in \mathbb{H}^{3} \text { and } \mathrm{u} \in \mathbb{H}^{1}
$$

such that $g \star \mathrm{T} \mapsto(g \star \mathrm{D}, g \star \mathrm{u})$.

\section{B. $S U(2)$-spaces of binary forms}

In this subsection the important link between the $\mathrm{SU}(2)$-space of binary forms and the $\mathrm{SO}(3)$-space of harmonic tensors will be pointed out. Through this correspondence it is possible to find polynomial invariants using classical invariant theory ${ }^{19}$. Most of the classical results presented in this subsection are borrowed from the classcial monograph of Sternberg ${ }^{36}$. Let us first consider the classical group morphism

$$
\varphi: \mathrm{SU}(2) \longrightarrow \mathrm{SO}(3)
$$

which kernel is $\{ \pm \mathbf{i d}\}$.

Now, let $\mathrm{S}_{2 k}$ be the space of $2 k$ th-degree binary forms over $\mathbb{C}^{2}$, meaning the $\mathbb{C}$-vector space ${ }^{52}$ 
of $\mathbf{f}$ given by

$$
\mathbf{f}(x, y):=\sum_{i=0}^{n}\left(\begin{array}{c}
2 k-i \\
i
\end{array}\right) a_{i} x^{2 k-i} y^{i} \text { for }(x, y) \in \mathbb{C}^{2}
$$

$\mathrm{SU}(2)$ has a natural irreducible action on the space $\mathrm{S}_{2 k}$, which is:

$$
(\gamma \cdot \mathbf{f})(x, y):=\mathbf{f}\left(\gamma^{-1} \cdot(x, y)\right) \text { for } \gamma \in \mathrm{SU}(2)
$$

Another important result states ${ }^{53}$ that there exists an isomorphism

$$
\psi: \mathrm{S}_{2 k} \longrightarrow \mathbb{H}^{k}
$$

satisfying

$$
\psi(\gamma \cdot \mathbf{f})=\varphi(\gamma) \star \psi(\mathbf{f})
$$

\section{Polynomial invariants on tensor spaces}

Let $\mathbb{T}$ be a stable $\mathrm{O}(3)$-tensor space and $\mathbb{C}[\mathbb{T}]$ the algebra of polynomials in $\mathbb{T}$. Now consider the following two invariant algebras

$$
\mathcal{A}:=\mathbb{C}[\mathbb{T}]^{\mathrm{O}(3)} \quad ; \quad \mathcal{A}^{s}:=\mathbb{C}[\mathbb{T}]^{\mathrm{SO}(3)}
$$

the first being the algebra of isotropic polynomials, while the second is the one of hemitropic polynomials. These algebras satisfy the following obvious inclusion:

$$
\mathcal{A} \subset \mathcal{A}^{s}
$$

As a graded algebra, $\mathcal{A}^{s}$ can be decomposed into $i$ th-degree homogeneous polynomials:

$$
\mathcal{A}^{s}=\mathcal{A}_{0}^{s} \oplus \mathcal{A}_{1}^{s} \oplus \cdots \oplus \mathcal{A}_{i}^{s} \cdots
$$

Hence

Lemma III.1. $\mathcal{A}$ is exactly the even part of $\mathcal{A}^{s}$; that is

$$
\mathcal{A}=\mathcal{A}_{0}^{s} \oplus \mathcal{A}_{2}^{s} \oplus \cdots \oplus \mathcal{A}_{2 i}^{s} \cdots
$$

Proof. It has to be observed that if $p$ is a $j$ th-degree homogeneous polynomial in $\mathcal{A}$, then

$$
p(-g \star \mathbf{T})=p(\mathbf{T})=(-1)^{j} p(g \star \mathbf{T})=(-1)^{j} p(\mathbf{T})
$$

for all $g \in \mathrm{SO}(3)$ and $\mathbf{T} \in \mathbb{T}$. This implies our lemma. 
This lemma allows to consider the algebra of $\mathrm{SO}(3)$-invariant polynomials on tensor spaces. Due to the isomorphism $\psi$ of III.1, this amount to consider the algebra of SU(2)-invariant polynomials on the space of binary forms. Once particularized to the space $\mathbb{H}^{3} \oplus \mathbb{H}^{1}$, the following result is obtained

Lemma III.2. The algebra of $\mathrm{SO}(3)$-invariant polynomials on the $\mathbb{C}$-vector space $\mathbb{H}^{3} \oplus \mathbb{H}^{1}$ is isomorphic to the algebra of $\mathrm{SU}(2)$-invariant polynomials on the $\mathbb{C}$-vector space $\mathrm{S}_{6} \oplus \mathrm{S}_{2}$.

As noted by Boehler et al. ${ }^{7}$, the algebra of $\mathrm{SO}(3)$-invariant polynomials on the real vector space $\mathbb{H}^{3} \oplus \mathbb{H}^{1}$ is isomorphic to the algebra of $\mathrm{SL}(2, \mathbb{C})$-invariant ${ }^{54}$ polynomials on the complex vector space $\mathrm{S}_{6} \oplus \mathrm{S}_{2}$; that is

$$
\mathbb{R}\left[\mathbb{H}^{3} \oplus \mathbb{H}^{1}\right]^{\mathrm{SO}(3)} \simeq \mathbb{C}\left[\mathrm{S}_{6} \oplus \mathrm{S}_{2}\right]^{\mathrm{SL}(2, \mathbb{C})}
$$

\section{Polynomial invariants of $\mathrm{S}_{6} \oplus \mathrm{S}_{2}$}

Let us consider the space $V:=\mathrm{S}_{6} \oplus \mathrm{S}_{2}$ of binary forms. In the monograph of Sturmfels ${ }^{37}$, some important and classical results about $\mathcal{R}:=\mathbb{C}[V]^{\mathrm{SL}(2, \mathbb{C})}$ can be found. These results provide important information to check whether a candidate basis of invariants generates or not the sought invariant algebra.

1. As a graded algebra, $\mathcal{R}$ can be decomposed

$$
\mathcal{R}=\mathcal{R}_{0} \oplus \mathcal{R}_{1} \oplus \cdots
$$

where each homogeneous space $\mathcal{R}_{i}$ is a finite $\mathbb{C}$-vector space. Let us consider the formal Hilbert series ${ }^{37}$

$$
H_{\mathcal{R}}(z):=\sum_{i} r_{i} z^{i}, \quad \text { with } r_{i}:=\operatorname{dim} \mathcal{R}_{i}
$$

2. In the case of binary forms, this series can be computed a priori. An integration approach $^{26}$ leads to the following result:

\section{Lemma III.3.}

$$
H_{\mathcal{R}}(z):=\frac{A(z)}{\left(1-z^{2}\right)\left(1-z^{4}\right)^{3}\left(1-z^{6}\right)^{2}\left(1-z^{10}\right)}
$$


where

$$
\begin{aligned}
A(z):= & 1+z^{2}+2 z^{4}+5 z^{6}+3 z^{7}+7 z^{8}+10 z^{9}+8 z^{10}+14 z^{11} \\
& +10 z^{12}+14 z^{13}+10 z^{14}+14 z^{15}+8 z^{16}+10 z^{17}+7 z^{18} \\
& +3 z^{19}+5 z^{20}+2 z^{22}+z^{24}+z^{26}
\end{aligned}
$$

3. By Hilbert's theorem ${ }^{21}$, the algebra $\mathcal{R}$ is finitely generated: there exist $p_{1}, p_{2}, \cdots, p_{n}$ such that

$$
\mathcal{R}=\mathbb{C}\left[p_{1}, p_{2}, \cdots, p_{n}\right]
$$

With the help of these results integrity bases can now be determined.

\section{INTEGRITY BASIS}

\section{A. Integrity basis for $\mathrm{S}_{6} \oplus \mathrm{S}_{2}$}

For binary forms, a classical way to construct covariants is to use the transvectant operator $^{30}$ :

Definition IV.1. Let $\mathbf{f}$ and $\mathbf{g}$ be two binary forms of respective order $m$ and $n$. We define the $r$ th-order transvectant of $\mathbf{f}$ and $\mathbf{g}$ to be the binary form:

$$
\{\mathbf{f}, \mathbf{g}\}_{r}:=\frac{(m-r) !}{m !} \times \frac{(n-r) !}{n !} \sum_{i=0}^{r}(-1)^{i}\left(\begin{array}{l}
r \\
i
\end{array}\right) \frac{\partial^{r} \mathbf{f}}{\partial^{r-i} x \partial^{i} y} \frac{\partial^{r} \mathbf{g}}{\partial^{i} x \partial^{r-i} y}
$$

As a first example, for a quadratic form $\mathbf{u} \in \mathrm{S}_{2}$ given by

$$
\mathbf{u}(x, y):=a_{0} x^{2}+2 a_{1} x y+a_{2} y^{2}
$$

we get

$$
\{\mathbf{u}, \mathbf{u}\}_{2}=2 a_{0} a_{2}-2 a_{1}^{2}
$$

which is a classical invariant. And for a cubic form $\mathrm{g} \in \mathrm{S}_{3}$ given by

$$
\mathbf{g}(x, y):=b_{0} x^{3}+3 b_{1} x^{2} y+3 b_{2} x y^{2}+b_{3} y^{3}
$$

we get a quadratic covariant:

$$
\{\mathbf{g}, \mathbf{g}\}_{2}=2\left(b_{0} b_{2}-b_{1}^{2}\right) x^{2}+2\left(b_{0} b_{3}-b_{1} b_{2}\right) x y+2\left(b_{1} b_{3}-b_{2}^{2}\right) y^{2}
$$


Such a covariant is said to be of degree 2 (in the coefficients $b_{i}$ ) and of order 2 (in the variables $x, y)$. This definition of degree and order is general: the degree of a covariant is the degree of the coefficients, while the order concerns the degree of the variables. Hence a 0th-order covariant is an invariant. The next computations will be made using the covariant basis for a sextic form given in table IV A. Such a basis is classic and has been computed by the end of nineteenth century ${ }^{19}$. In this table covariants of degree $d$ and order $o$ are denoted $\mathbf{C}_{d, o}$.

\begin{tabular}{|c|c|c|c|c|c|c|c|}
\hline $\mathrm{d} / \mathrm{o}$ & 0 & 2 & 4 & 6 & 8 & 10 & 12 \\
\hline 1 & & & & f & & & \\
\hline 2 & $\{\mathbf{f}, \mathbf{f}\}_{6}$ & & $\{\mathbf{f}, \mathbf{f}\}_{4}$ & & $\{\mathbf{f}, \mathbf{f}\}_{2}$ & & \\
\hline 3 & & $\left\{\mathbf{C}_{2,4}, \mathbf{f}\right\}_{4}$ & & $\left\{\mathbf{C}_{2,4}, \mathbf{f}\right\}_{2}$ & $\left\{\mathbf{C}_{2,4}, \mathbf{f}\right\}_{1}$ & & $\left\{\mathbf{C}_{2,8}, \mathbf{f}\right\}_{1}$ \\
\hline 4 & $\left\{\mathbf{C}_{2,4}, \mathbf{C}_{2,4}\right\}_{4}$ & & $\left\{\mathbf{C}_{3,2}, \mathbf{f}\right\}_{2}$ & $\left\{\mathbf{C}_{3,2}, \mathbf{f}\right\}_{1}$ & & $\left\{\mathbf{C}_{2,8}, \mathbf{C}_{2,4}\right\}_{1}$ & \\
\hline 5 & & $\left\{\mathbf{C}_{2,4}, \mathbf{C}_{3,2}\right\}_{2}$ & $\left\{\mathbf{C}_{2,4}, \mathbf{C}_{3,2}\right\}_{1}$ & & $\left\{\mathbf{C}_{2,8}, \mathbf{C}_{3,2}\right\}_{1}$ & & \\
\hline 6 & $\left\{\mathbf{C}_{3,2}, \mathbf{C}_{3,2}\right\}_{2}$ & & & $\begin{aligned} \mathbf{C}_{6,6 a} & :=\left\{\mathbf{C}_{3,8}, \mathbf{C}_{3,2}\right\}_{2} \\
\mathbf{C}_{6,6 b} & :=\left\{\mathbf{C}_{3,6}, \mathbf{C}_{3,2}\right\}_{1}\end{aligned}$ & & & \\
\hline 7 & & $\left\{\mathbf{f}, \mathbf{C}_{3,2}^{2}\right\}_{4}$ & $\left\{\mathbf{f}, \mathbf{C}_{3,2}^{2}\right\}_{3}$ & & & & \\
\hline 8 & & $\left\{\mathbf{C}_{2,4}, \mathbf{C}_{3,2}^{2}\right\}_{3}$ & & & & & \\
\hline 9 & & & $\left\{\mathbf{C}_{3,8}, \mathbf{C}_{3,2}^{2}\right\}_{4}$ & & & & \\
\hline 10 & $\left\{\mathbf{C}_{3,2}^{3}, \mathbf{f}\right\}_{6}$ & $\left\{\mathbf{C}_{3,2}^{3}, \mathbf{f}\right\}_{5}$ & & & & & \\
\hline 12 & & $\left\{\mathbf{C}_{3,8}, \mathbf{C}_{3,2}^{3}\right\}_{6}$ & & & & & \\
\hline 15 & $\left\{\mathbf{C}_{3,8}, \mathbf{C}_{3,2}^{4}\right\}_{8}$ & & & & & & \\
\hline
\end{tabular}

TABLE I. Covariant basis for $\mathrm{S}_{6}$

The following result ${ }^{19,29}$ is used to determine a finite generating set of invariants for the algebra $\mathcal{R}$ :

Theorem IV.2. If $\left\{\mathbf{h}_{1}, \cdots, \mathbf{h}_{s}\right\}$ is a covariant basis for $\mathrm{S}_{n}$, and if $\mathbf{u}$ is a quadratic form, then irreducible invariants of $\mathrm{S}_{n} \oplus \mathrm{S}_{2}$ are taken from one of this set:

- $\left\{\mathbf{h}_{i}, \mathbf{u}^{r}\right\}_{2 r}$ for $i=1 \cdots s$ such that $\mathbf{h}_{i}$ is of order $2 r$;

- $\left\{\mathbf{h}_{i} \mathbf{h}_{j}, \mathbf{u}^{r}\right\}_{2 r}$ where $\mathbf{h}_{i}$ is of order $2 p+1$ and $\mathbf{h}_{j}$ is of order $2 r-2 p-1$. 
It should be noted that the obtained generating set need not be irreducible. Hence, invariants can be obtained, degree per degree:

- Degree 2:

$$
i_{2}:=\{\mathbf{f}, \mathbf{f}\}_{6} j_{2}:=\{\mathbf{u}, \mathbf{u}\}_{2}
$$

- Degree 4:

$$
\begin{aligned}
& i_{4}:=\left\{\mathbf{C}_{2,4}, \mathbf{C}_{2,4}\right\}_{4} j_{4}:=\left\{\mathbf{C}_{3,2}, \mathbf{u}\right\}_{2} \\
& k_{4}:=\left\{\mathbf{C}_{2,4}, \mathbf{u}^{2}\right\}_{4} \quad l_{4}:=\left\{\mathbf{f}, \mathbf{u}^{3}\right\}_{6}
\end{aligned}
$$

- Degree 6:

$$
\begin{aligned}
& i_{6}:=\left\{\mathbf{C}_{3,2}, \mathbf{C}_{3,2}\right\}_{2} j_{6}:=\left\{\mathbf{C}_{5,2}, \mathbf{u}\right\}_{2} \quad k_{6}:=\left\{\mathbf{C}_{4,4}, \mathbf{u}^{2}\right\}_{4} \\
& l_{6}:=\left\{\mathbf{C}_{3,6}, \mathbf{u}^{3}\right\}_{6} \quad m_{6}:=\left\{\mathbf{C}_{2,8}, \mathbf{u}^{4}\right\}_{8}
\end{aligned}
$$

- Degree 7:

$$
i_{7}:=\left\{\mathbf{C}_{5,4}, \mathbf{u}^{2}\right\}_{4} j_{7}:=\left\{\mathbf{C}_{4,6}, \mathbf{u}^{3}\right\}_{6} k_{7}:=\left\{\mathbf{C}_{3,8}, \mathbf{u}^{4}\right\}_{8}
$$

- Degree 8:

$$
i_{8}:=\left\{\mathbf{C}_{7,2}, \mathbf{u}\right\}_{2}
$$

- Degree 9:

$$
\begin{aligned}
& i_{9}:=\left\{\mathbf{C}_{8,2}, \mathbf{u}\right\}_{2} \quad j_{9}:=\left\{\mathbf{C}_{7,4}, \mathbf{u}^{2}\right\}_{4} \quad k_{9}:=\left\{\mathbf{C}_{6,6 a}, \mathbf{u}^{3}\right\}_{6} \\
& l_{9}:=\left\{\mathbf{C}_{6,6 b}, \mathbf{u}^{3}\right\}_{6} \quad m_{9}:=\left\{\mathbf{C}_{5,8}, \mathbf{u}^{4}\right\}_{8} n_{9}:=\left\{\mathbf{C}_{4,10}, \mathbf{u}^{5}\right\}_{10} \\
& o_{9}:=\left\{\mathbf{C}_{3,12}, \mathbf{u}^{6}\right\}_{12}
\end{aligned}
$$

- Degree 10:

$$
i_{10}:=\left\{\mathbf{C}_{3,2}^{3}, \mathbf{f}\right\}_{6}
$$

- Degree 11:

$$
i_{11}:=\left\{\mathbf{C}_{9,4}, \mathbf{u}^{2}\right\}_{4} \quad j_{11}:=\left\{\mathbf{C}_{10,2}, \mathbf{u}\right\}_{2}
$$

- Degree 13:

$$
i_{13}:=\left\{\mathbf{C}_{12,2}, \mathbf{u}\right\}_{2}
$$


- Degree 15:

$$
i_{15}:=\left\{\mathbf{C}_{3,8}, \mathbf{C}_{3,2}^{4}\right\}_{8}
$$

By use of theorem IV.2 we know that $\mathcal{R}=\mathbb{C}\left[i_{2}, j_{2}, \cdots, i_{15}\right]$. Now we compute homogeneous space $\operatorname{dimensions} \operatorname{dim}\left(\mathcal{R}_{i}\right)_{i=1 \ldots 15}$ and compare them with the $r_{i}$ of the Hilbert series $H_{\mathcal{R}}$. These computations have been performed using scripts written in Macaulay $2^{20}$, the following result is obtained:

Proposition IV.3. A minimal Hilbert basis for the algebra $\mathbb{C}\left[\mathrm{S}_{6} \oplus \mathrm{S}_{2}\right]^{\mathrm{SL}_{2}}$ is given by the 27 invariants

$\begin{array}{cccc}\text { Name } & \text { Degree } & \text { Name } & \text { Degree } \\ i_{2}, j_{2} & 2 & i_{9}, j_{9}, k_{9}, l_{9}, m_{9}, n_{9}, o_{9} & 9 \\ i_{4}, j_{4}, k_{4}, l_{4} & 4 & i_{10} & 10 \\ i_{6}, j_{6}, k_{6}, l_{6}, m_{6}, & 6 & i_{11}, j_{11} & 11 \\ i_{7}, j_{7}, k_{7} & 7 & i_{13} & 13 \\ i_{8} & 8 & i_{15} & 15\end{array}$

\section{B. Integrity basis for $\mathbb{H}^{3} \oplus \mathbb{H}^{1}$}

In order to obtain an integrity basis for $\mathbb{H}^{3} \oplus \mathbb{H}^{1}$, the even part of $\mathbb{C}\left[\mathrm{S}_{6} \oplus \mathrm{S}_{2}\right]^{\mathrm{SL}_{2}}$ has to be determined. For that purpose, we consider the algebra

$$
\mathcal{B}:=\mathbb{C}\left[i_{2}, j_{2}, i_{4}, j_{4}, k_{4}, l_{4}, i_{6}, j_{6}, k_{6}, l_{6}, m_{6}, i_{8}, i_{10}\right]
$$

and compute $\operatorname{dim}\left(\mathcal{B}_{2 j}\right)_{j=1 \ldots 13}$ to compare it with the $r_{2 j}$ of the Hilbert series $H_{\mathcal{R}}$. Finally we get:

Lemma IV.4. The even part of the algebra $\mathbb{C}\left[\mathrm{S}_{6} \oplus \mathrm{S}_{2}\right]^{\mathrm{SL}_{2}}$ is generated by the thirteen invariants

$$
i_{2}, j_{2}, i_{4}, j_{4}, k_{4}, l_{4}, i_{6}, j_{6}, k_{6}, l_{6}, m_{6}, i_{8}, i_{10}
$$

Now, from lemmas III.1 and IV.4 and using the isomorphism $\psi$ of III.1:

Theorem IV.5. There exist polynomials $A_{2}, B_{2}$ of degree $2, A_{4}, B_{4}, C_{4}, D_{4}$ of degree 4 , $A_{6}, B_{6}, C_{6}, D_{6}, E_{6}$ of degree $6, A_{8}$ of degree 8 and $A_{10}$ of degree 10 such that

$$
\mathbb{C}\left[\mathbb{H}^{3} \oplus \mathbb{H}^{1}\right]^{\mathrm{O}(3)}=\mathbb{C}\left[A_{2}, B_{2}, \ldots, A_{10}\right]
$$


In other terms,

Theorem IV.6. An integrity basis for $\mathbb{R}\left[\mathbb{H}^{3} \oplus \mathbb{H}^{1}\right]^{\mathrm{O}(3)}$ is given by:

$$
\begin{array}{lll}
I_{2}:=\mathrm{D}_{i j k} \mathrm{D}_{i j k} & J_{2}:=\mathrm{u}_{i}^{2} & I_{4}:=\mathrm{D}_{i j k} \mathrm{D}_{i j l} \mathrm{D}_{p q k} \mathrm{D}_{p q l} \\
J_{4}:=\mathrm{D}_{i j k} \mathrm{u}_{k} \mathrm{D}_{i j l} \mathrm{u}_{l} & K_{4}:=\mathrm{D}_{i j k} \mathrm{D}_{i j l} \mathrm{D}_{k l p} \mathrm{u}_{p} & L_{4}:=\mathrm{D}_{i j k} \mathrm{u}_{k} \mathrm{u}_{j} \mathrm{u}_{i} \\
I_{6}:=\mathrm{v}_{i}^{2} & J_{6}:=\mathrm{D}_{i j k} \mathrm{D}_{i j l} \mathrm{u}_{k} \mathrm{D}_{l p q} \mathrm{u}_{p} \mathrm{u}_{q} & K_{6}:=\mathrm{v}_{k} \mathrm{w}_{k} \\
L_{6}:=\mathrm{D}_{i j k} \mathrm{D}_{i j l} \mathrm{D}_{k} v_{l} & M_{6}:=\mathrm{D}_{i j k} \mathrm{D}_{p q k} \mathrm{u}_{i} \mathrm{u}_{j} \mathrm{u}_{p} \mathrm{u}_{q} & I_{8}:=\mathrm{D}_{i j k} \mathrm{D}_{i j l} \mathrm{u}_{k} \mathrm{D}_{p q l} \mathrm{D}_{p q r} \mathrm{v}_{r} \\
I_{10}:=\mathrm{D}_{i j k} \mathrm{v}_{i} \mathrm{v}_{j} \mathrm{v}_{k} & &
\end{array}
$$

in which

$$
\mathrm{v}_{p}:=\mathrm{D}_{i j k} \mathrm{D}_{i j l} \mathrm{D}_{k l p} ; \mathrm{w}_{k}:=\mathrm{D}_{i j k} \mathrm{u}_{i} \mathrm{u}_{j}
$$

Proof. Let define

$$
\mathcal{B}:=\mathbb{C}\left[I_{2}, J_{2}, \cdots, I_{10}\right] \text { and } \mathcal{A}:=\mathbb{C}[V]^{\mathrm{O}(3)}=\mathbb{C}\left[I_{2}, B_{2}, \cdots, A_{10}\right]
$$

We put $\mathcal{B}_{k}\left(\right.$ resp. $\left.\mathcal{A}_{k}\right)$ to be the space of $k$ th-degree homogeneous space of $\mathcal{B}$ (resp. $\mathcal{A}$ ). Now it is clear that

$$
\mathcal{B} \subset \mathcal{A}
$$

By computing dimensions of the vector spaces $\mathcal{B}_{k}$ up to $k=10$ the same dimension as $\mathcal{A}_{k}$ are obtained. Thus each generator $A_{2}, B_{2}, \cdots, A_{10}$ belongs to $\mathcal{B}$. Hence it can be concluded that $\mathcal{A}=\mathcal{B}$. Now, because all invariants $I_{2}, \cdots, I_{10}$ have real coefficients, this leads us to an integrity basis for $\mathbb{R}\left[\mathbb{H}^{3} \oplus \mathbb{H}^{1}\right]^{\mathrm{O}(3)}$.

\section{ACKNOWLEDGMENTS}

The second author wishes to thank the pole EMC2 and the Region Pays de la Loire for their funding.

\section{REFERENCES}

${ }^{1}$ N. Auffray and B. Kolev and M. Petitot. On anisotropic polynomial relations for the elasticity tensor. J. Elast., 1-27,2013.

${ }^{2} \mathrm{~N}$. Auffray. On the algebraical structure of isotropic generalized elasticity theories. Math. \& Mech. of Solids, 2014,(On-line First). 
${ }^{3}$ G. Backus. A geometrical picture of anisotropic elastic tensors. Rev. Geophys., 8(3):633671,1970 .

${ }^{4}$ L. Bedratyuk. A complete minimal system of covariants for the binary form of degree 7 . J. Symbolic Comput., 44(2):211-220, 2009.

${ }^{5}$ J.-P. Boehler. On irreducible representations for isotropic scalar functions. ZAMM Z. Angew. Math. Mech., 57:323-327, 1977.

${ }^{6}$ J.-P. Boehler. Application of tensor functions in solid mechanics. CISM Courses and Lectures. Springer-Verlag, Wien, 1987.

${ }^{7}$ J.-P. Boehler, A. A. Kirillov, Jr., and E. T. Onat. On the polynomial invariants of the elasticity tensor. J. Elasticity, 34(2):97-110, 1994.

${ }^{8}$ A.E. Brouwer and M. Popoviciu. The invariants of the binary decimic. J. Symbolic Comput., 45(8):837-843, 2010.

${ }^{9}$ A.E. Brouwer and M. Popoviciu. The invariants of the binary nonic. J. Symbolic Comput., 45(6):709-720, 2010.

${ }^{10}$ F. dell'Isola, G. Sciarra, and S. Vidoli. Generalized Hooke's law for isotropic second gradient materials. Proc. R. Soc. A, 465:2177-2196, 2009.

${ }^{11}$ H. Derksen and G. Kemper. Computational Invariant Theory. Springer, 2002.

${ }^{12}$ J. Dixmier and D. Lazard. Le nombre minimum d'invariants fondamentaux pour les formes binaires de degré 7. Portugal. Math., 43(3):377-392, 1985.

${ }^{13}$ M. Ferretti, A. Madeo, F. dell'Isola, and P. Boisse. Modeling the onset of shear boundary layers in fibrous composite reinforcements by second-gradient theory. ZAMP Z. Angew. Math. Phys., 2013 (On-Line First).

${ }^{14}$ S. Forest and R. Sievert. Nonlinear microstrain theories. Int. J. Solids. Struct., 43:7224$7245,2006$.

${ }^{15}$ S. Forte and M. Vianello. Symmetry classes for elasticity tensors. J. Elasticity, 43(2):81-108, 1996.

${ }^{16} \mathrm{~S}$. Forte and M. Vianello. Functional bases for transversely isotropic and transversely hemitropic invariants of elasticity tensors. Int. J. Eng. Sci., 51:543-552, 1998.

${ }^{17}$ F. Gronwald and F.W. Hehl. Stress and hyperstress as fundamental concepts in continuum mechanics and in relativistic field theory. arXiv preprint gr-qc/9701054, 1997.

${ }^{18} \mathrm{P}$. Gordan. Beweis, dass jede covariante und invariante einer bineren form eine ganze func- 
tion mit numerischen coefficienten einer endlichen anzahl solcher formen ist. Journal fur die reine und angewandte Mathematik, page 323-354, 1868.

${ }^{19}$ J.H. Grace and A. Young. The algebra of invariants. Cambridge Library Collection. Cambridge University Press, Cambridge, 2010.

${ }^{20}$ D.R. Grayson and M.E. Stillman. Macaulay2, a software system for research in algebraic geometry. http://www.math.uiuc.edu/Macaulay2/.

${ }^{21}$ D. Hilbert. Theory of algebraic invariants. Cambridge University Press, Cambridge, 1993.

${ }^{22}$ J. Jerphagnon, D. Chemla, and R. Bonneville. The description of the physical properties of condensed matter using irreducible tensors. Adv. Phys., 27:609-650, 1978.

${ }^{23}$ A. Javili and F. dell'Isola and P. Steinmann. Geometrically nonlinear higher-gradient elasticity with energetic boundaries. J. Mech. Phys. Solids., 61:2381-2401, 2013.

${ }^{24}$ E. Kiral and G. F. Smith. On the constitutive relations for anisotropic materials triclinic, monoclinic, rhombic, tetragonal and hexagonal crystal systems. Int. J. Eng. Sci., 12:471490, 1974.

${ }^{25}$ H. Le Quang and Q.-C. He. The number and types of all possible rotational symmetries for flexoelectric tensors. 467(2132):2369-2386, 2011.

${ }^{26} \mathrm{P}$. Littelmann and C. Procesi. On the Poincaré series of the invariants of binary forms. J. Algebra, 133(2):490-499, 1990.

${ }^{27}$ I. Liu. On representations of anisotropic invariants. Int. J. Eng. Sci., 20:1099-1109, 1982.

${ }^{28}$ R.D. Mindlin. Micro-structure in linear elasticity. Arch. Rational Mech. Anal., 16(1):51-78, 1964.

${ }^{29}$ M. Olive. On Gordan's algorithm for binary forms. (To be published).

${ }^{30}$ P.J. Olver. Classical invariant theory, volume 44 of London Mathematical Society Student Texts. Cambridge University Press, Cambridge, 1999.

${ }^{31}$ V.L. Popov and E.B. Vinberg. Invariant theory. Springer Berlin Heidelberg, 1994.

${ }^{32}$ T. Shioda. On the graded ring of invariants of binary octavics. Amer. J. Math., 89:10221046, 1967.

${ }^{33}$ G.F. Smith and G. Bao. Isotropic invariants of traceless symmetric tensors of orders three and four. Int. J. Eng. Sci., 35(15):1457-1462, 1997.

${ }^{34}$ G.F. Smith. Constitutive Equations for Anisotropic and Isotropic Materials. North-Holland, Amsterdam, 1994. 
${ }^{35}$ A.J.M. Spencer. A note on the decomposition of tensors into traceless symmetric tensors. Int. J. Eng. Sci. , 8:475-481, 1970.

${ }^{36}$ S. Sternberg. Group theory and physics. Cambridge University Press, Cambridge, 1994.

${ }^{37}$ B. Sturmfels. Algorithms in invariant theory. Texts and Monographs in Symbolic Computation. Springer, second edition, 2008.

${ }^{38}$ R.A. Toupin. Elastic materials with couple-stresses. Arch. Rational Mech. Anal., 11(1):385414, 2012.

${ }^{39} \mathrm{~A}$. Thionnet and Ch. Martin. A new constructive method using the theory of invariants to obtain behavior laws, Int. J. Solids. Struct., 43:325-345, 2006.

${ }^{40} \mathrm{~A}$. Wineman and A. Pipkin. Material symmetry restrictions on constitutive equations. Arch. Rational Mech. Anal., 17:184-214, 1964.

${ }^{41}$ Q.-S. Zheng. Theory of representations for tensor functions - a unified invariant approach to constitutive equations. Arch. Appl. Mech., 47:545-587, 1994.

${ }^{42}$ Q.-S. Zheng and J. Betten. On the tensor function representation of 2nd-order and 4th-order tensors. part i. ZAMM Z. Angew. Math. Mech., 75:269-281, 1995.

${ }^{43}$ This is due to the Hilbert's theorem which states that for any finite set of tensors a finite integrity basis exists.

${ }^{44} \mathrm{It}$ is worth noting that the definition of an integrity basis is not restricted to $\mathrm{O}(3)$, nor restricted to real vector spaces.

${ }^{45}$ Generally the group action is not $\mathrm{O}(3)$, but $\mathrm{O}(2)$ (or a subgroup) ${ }^{16,24}$, or $\mathrm{O}(6)$ (or a subgroup) $)^{6,42}$.

${ }^{46}$ For low-order tensors this distinction is also true but less relevant.

${ }^{47}$ The case of an $\mathrm{O}(3)$-action is closely related to the $\mathrm{SO}(3)$ case.

${ }^{48}$ In which, the Einstein summation convention over repeated indices has been used.

${ }^{49}$ Meaning they have a null laplacian.

${ }^{50}$ The empty space and the total space are always stable.

${ }^{51}$ The explicit expression of the isomorphism has been given, for example, by Auffray ${ }^{2}$.

${ }^{52}$ It has to be noted that $\mathrm{S}_{2 k}$ is a $2 k+1 \mathbb{C}$-vector space.

${ }^{53}$ This result is a direct consequence of the Schur lemma, a classical result of group representation.

${ }^{54} \mathrm{SL}(2, \mathbb{C})$ is the complexification of $\mathrm{SU}(2)$. 